International Journal of Medical Anesthesiology 2021; 4(1): 14-19

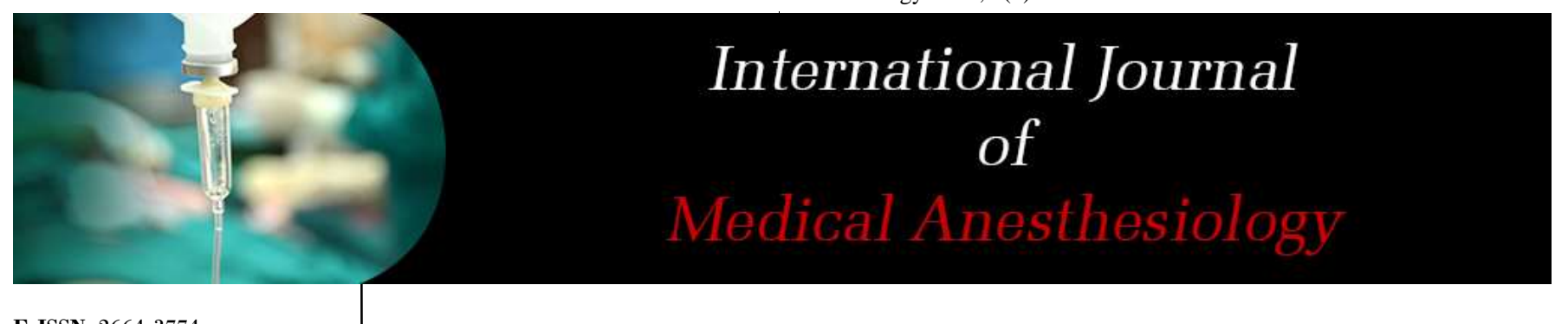

E-ISSN: 2664-3774

P-ISSN: 2664-3766 www.anesthesiologypaper.com IJMA 2021; 4(1): 14-19

Received: 03-11-2020

Accepted: 12-12-2020

Dr. Shwethapriya $\mathbf{R}$ Associate Professor, Department of Critical Care, KMC Manipal, MAHE University, Karnataka, India

Dr. Manjunath Prabhu Professor, Department of Anesthesiology, KMC Manipal, MAHE University, Karnataka, India

Dr. Souvik Chaudhury Assistant Professor, Department of Critical Care, KMC Manipal, MAHE University, Karnataka, India

Corresponding Author: Dr. Shwethapriya $\mathbf{R}$ Associate Professor, Department of Critical Care, KMC Manipal, MAHE University, Karnataka, India

\section{A comparison of blind and lightwand guided tracheal intubation through the intubating laryngeal mask airway}

\author{
Dr. Shwethapriya R, Dr. Manjunath Prabhu and Dr. Souvik Chaudhury
}

DOI: https://doi.org/10.33545/26643766.2021.v4.i1a.189

\begin{abstract}
The purpose of our study was to compare blind versus lightwand guided technique of intubation through the ILMA with respect to success of intubation, time taken for successful intubation and incidence of pharyngolaryngeal morbidity. A written and informed consent for participation in the study was obtained from the patients selected. Patients were kept nil per oral as per the standard protocol. Patients were randomly allocated to one of two equal sized groups ( $n=30$ each). A random number table was used for randomization and they were divided to two groups. Intubation through the ILMA was successful in all 60 patients involved in the study, with an overall success rate of $100 \%$. There was no statistical difference in the median intubation time between the two groups as determined by the Mann Whitney U test. However, intubation times in the blind group had a skewed distribution as indicated by the large standard deviation. Whereas, the lightwand group had intubation times closer to the mean.
\end{abstract}

Keywords: Blind and lightwand, tracheal intubation, laryngeal mask airway

\section{Introduction}

Airway management is a vital skill required for the anaesthesiologists to successfully manage a case of anticipated or unanticipated difficult airway ${ }^{[1]}$. Several airway devices and techniques can be employed in such situations. Intubating laryngeal mask airway and the illuminated stylet are two such devices which have gained popularity for management of difficult airways ${ }^{[2]}$. The purpose of our study was to compare blind versus lightwand guided technique of intubation through the ILMA with respect to success of intubation, time taken for successful intubation and incidence of pharyngolaryngeal morbidity.

We conducted this study to evaluate the usefulness of the flexible lightwand as an aid for intubation through the ILMA. We compared the success rate, the time for intubation and the number of adjustment manoeuvres required to achieve successful intubation, with the blind technique of intubation through the ILMA. We also assessed the incidence of postoperative complications such as sore throat and hoarseness of voice.

\section{Methodology}

The study was a prospective, randomized, controlled trial. Following the ethical committee approval, sixty adult patients, aged between 18 to 65 years of ASA physical status I or II, weighing between 30 to $70 \mathrm{kgs}$, scheduled for elective surgery requiring general anaesthesia and endotracheal intubation were enrolled for the study. Patients with known or predicted difficult airway, diseases of the respiratory system, history of sore throat in the last 10 days, known gastro-esophageal reflux disease or other esophageal pathology, body mass index > $30 \mathrm{~kg} \mathrm{~m}^{-2}$, ischaemic heart disease, cerebrovascular disease and intracranial mass lesions, history suggestive of obstructive sleep apnoea and conditions requiring rapid sequence induction were excluded from the study.

A written and informed consent for participation in the study was obtained from the patients selected. Patients were kept nil per oral as per the standard protocol.

Patients were randomly allocated to one of two equal sized groups ( $n=30$ each). A random number table was used for randomization and they were divided to one of the following two groups.

1. Blind: patients were blindly intubated through the ILMA (LMA Fastrach ${ }^{\mathrm{TM}}$, Laryngeal MASK Company Limited, Henley-on-Thames, UK). 
2. Lightwand: Trachlight ${ }^{\mathrm{TM}}$ (Laryngeal Medical Corporation, New York, USA), without the inner metal stylet, was used as a guide for intubation through the ILMA.

The study was conducted by three observers: Observer 1 was a consultant anaesthesiologist with prior experience with both the ILMA and the Trachlight ${ }^{\mathrm{TM}}$ lightwand who performed all the intubations in the study. Observer 2 was a postgraduate student in anaesthesiology who did the preanaesthetic assessment of all the patients and recorded all the parameters. Observer 3, postgraduate student in anaesthesiology who was blinded to the technique of intubation, followed up all the patients 18 to 24 hours post operatively and collected data on sore throat and hoarseness of voice.

On entering the operating room, intravenous access was secured and monitors were connectd. The patient was made to lie supine with the head in the neutral position supported by a standard pillow.

The patient was preoxygenated. At the start of preoxygenation, the patient was administered $1 \mathrm{mcg} / \mathrm{kg}$ fentanyl and $0.2 \mathrm{mg}$ glycopyrrolate. Anaesthesia was induced with propofol and neuromuscular blockade was achieved with vecuronium bromide. After disappearance of a response to the train of four stimulation, ILMA insertion was attempted.

The ILMA (LMA Fastrach, Laryngeal Mask Company Limited, Henley-on-Thames, UK) after lubrication with a water soluble lubricant was inserted as per the manufacturer's guidelines, with a single-handed rotational technique with the patient's head in neutral position.

A size 3 ILMA was used for patients with a body weight < $50 \mathrm{~kg}$, and a size 4 ILMA for patients with a body weight of $\geq 50 \mathrm{~kg}$. Once inserted, the cuff was inflated with air: the size 3 ILMA with $20 \mathrm{ml}$ of air, and the size 4 ILMA with 30 $\mathrm{ml}$ of air.

The anaesthesia circuit was connected to the ILMA, and the ability to mask ventilate through the ILMA was checked by confirming bilateral chest expansion and appearance of a capnographic trace. If ventilation through the ILMA was inadequate, its position was adjusted until an optimal seal was achieved, as confirmed by adequate filling of the bag and ability to ventilate gently. The position of the ILMA was maintained for intubation by holding its handle. If adequate ventilation could not be achieved with the ILMA, then such a patient was excluded from the study.

All patients were intubated with the straight, wirereinforced, cuffed, silicone tube (TT) with a curved tip, specially designed for use with the ILMA. A size $7.0 \mathrm{~mm}$ internal diameter TT was used for all patients. A time limit of 3 minutes was allocated to achieve successful intubation once the circuit was disconnected from the ILMA.

In the blind group, the silicone TT, after adequate lubrication, was inserted into the ILMA till the $15 \mathrm{~cm}$ mark on the TT. This indicated that the TT tip was beyond the epiglottic elevator bar. Intubation was then attempted by gently advancing the TT. If resistance was encountered to its passage, the TT was pulled back into the ILMA, to the $15 \mathrm{~cm}$ mark, and a series of realignment manoeuvers were performed in sequence.

Extension manoeuver: the handle of the ILMA was pulled back towards the intubator.

Up-down manoeuver: the ILMA was withdrawn by $5 \mathrm{~cm}$ and then reinserted without deflating its cuff.
Chandy manoeuver: This involved two steps; First step: rotation of ILMA in the sagittal plane. Second step: the ILMA had to be lifted by the handle by 2 to $5 \mathrm{~mm}$ in the sagittal plane and maintained in this position during intubation attempts.

After each adjustment manoeuver, attempts to advance the TT were made. After successful advancement of the TT into the trachea, its cuff was inflated with 5 to $6 \mathrm{ml}$ of air. The breathing circuit was then connected to the TT and its position confirmed (vide infra).

In the Lightwand group, the silicone TT, was mounted on the lubricated flexible Trachlight lightwand without the inner metal guide, such that the lighted tip of the lightwand came to lie just short of the beveled tip of the TT. This constituted the TT-lightwand assembly.

After successful insertion of the ILMA as described above, the TT-lightwand assembly was introduced into the ILMA. The lightwand was switched on after $15 \mathrm{~cm}$ of the TT had been passed into the ILMA.

The lights in the operating room were dimmed, and a glow over the anterior part of the neck was looked for. The detection of a distinct glow of light without a halo at the cricothyroid membrane was taken as evidence of proper alignment of the TT with the laryngeal inlet. At this point, further advancement of the TT was attempted.

If the glow was seen in the lateral aspect of the neck or resistance was felt to its passage, then the adjusting manoeuvers previously described were applied to the ILMA. If no resistance was felt, the TT was advanced adequately and its cuff was inflated. The lightwand was disengaged from the TT and removed. The breathing circuit was then connected to the TT.

In both groups, correct placement of the TT was confirmed by appearance of a capnographic trace suggestive of tracheal intubation. Once confirmed, the ILMA was removed using a stabilizing rod after deflation of the ILMA cuff.

If the patient showed signs of a lighter plane of anaesthesia any time during the procedure, intravenous propofol $20 \mathrm{mg}$ bolus was given.

When the anaesthesia circuit was connected to the TT, after it was assumed the TT was successfully advanced into the trachea, an attempt of intubation was considered to be completed.

Intubation time was defined as the time between disconnection of the circuit from the ILMA to reconnection of the circuit to the TT and appearance of a capnographic trace.

The absence of a capnographic trace was diagnosed as an oesophageal intubation. The TT was then withdrawn and further attempts were made to reintubate using the technique specific to the group. This was permitted only if the 3 minute time period had not elapsed.

Once the circuit was disconnected from the ILMA for intubation, it was to be reconnected only in case of a desaturation to less than $95 \%$. Thus attempts at intubation could proceed uninterrupted up to the time limit of 3 minutes. A failed attempt was defined as failure to intubate within 3 minutes after disconnection of the circuit from the ILMA or desaturation to less than $95 \%$ or failure of all three adjustment manoeuvres. If intubation through the ILMA failed, then such a patient was to be intubated using direct laryngoscopy with an appropriate sized Macintosh blade. The laryngoscopic grade was to be assessed as per the 
Cormack and Lehane score.

After successful intubation, further anaesthesia management was continued as planned by the anaesthesia team assigned for that particular case.

Patients were followed up 18 to 24 hours post-operatively by the third observer for evidence of a sore throat or hoarseness of voice.

The sore throat or hoarseness of voice was graded as follows:

Sore throat was graded as:

A. None: no sore throat

B. Mild: less severe than with a cold

C. Moderate: similar to that noted with a cold

D. Severe: more severe than with a cold

Hoarseness was graded as:

A. None: no hoarseness

B. Mild: noted by the patient

C. Moderate: obvious to observer

D. Severe: aphonia

\section{Statistical analysis of collected data}

Student's unpaired $t$ test was utilized for continuous variables where applicable. Chi square test, Fisher's exact test and Mann Whitney U test were applied as considered appropriate.

$\mathrm{p}$ value $<0.05$ was considered as statistically significant.

\section{Results}

In all 60 patients, the ILMA was successfully inserted on the first attempt. The time for insertion of the ILMA in both groups was comparable as shown in table 1.

Table 1: ILMA insertion time and success rate

\begin{tabular}{|c|c|c|c|}
\hline Parameter & $\begin{array}{c}\text { Blind group } \\
(\mathbf{n = 3 0})\end{array}$ & $\begin{array}{c}\text { Lightwand } \\
\text { group }(\mathbf{n = 3 0})\end{array}$ & $\begin{array}{c}\mathbf{p} \\
\text { value }\end{array}$ \\
\hline $\begin{array}{c}\text { Insertion time }(\mathrm{s}) \\
\text { Mean } \pm \text { S.D }\end{array}$ & $12.33 \pm 2.20$ & $11.93 \pm 2.51$ & $0.514 *$ \\
\hline Success on $1^{\text {st }}$ attempt & 30 & 30 & \\
\hline
\end{tabular}

*Student's unpaired $t$ test

Intubation through the ILMA was successful in all 60 patients involved in the study, with an overall success rate of $100 \%$. There was no statistical difference in the median intubation time between the two groups as determined by the Mann Whitney U test. However, intubation times in the blind group had a skewed distribution as indicated by the large standard deviation. Whereas, the lightwand group had intubation times closer to the mean.

The overall first attempt success rate for intubation through the ILMA was $90 \%$. First attempt success rate was $100 \%$ in the lightwand group and $80 \%$ in the blind group. The difference between the two groups with respect to first attempt success rate was statistically significant with a $p$ value of 0.02 .

Table 2: Time taken for intubation through the ILMA, and success rate at each attempt

\begin{tabular}{|c|c|c|c|}
\hline Parameter & Blind n=30 & Lightwand n=30 & p value \\
\hline Intubation time (s) & & & \\
\hline Mean \pm S.D & $26.73 \pm 21.09$ & $20.7 \pm 5.68$ & $0.438^{*}$ \\
\hline median $\}$ & $\{20.5\}$ & $\{20.0\}$ & \\
\hline [interquartile range] $^{\text {st }}$ attempt & $24.75]$ & {$[7.25]$} & $0.02^{\star}$ \\
\hline Success on $1^{\text {st }}$ & 30 & \\
\hline Success on 2 ${ }^{\text {nd }}$ attempt & 5 & 0 & \\
\hline Success on 3 ${ }^{\text {rd }}$ attempt & 1 & 0 & \\
\hline
\end{tabular}

* Mann Whitney U test

- Fisher's exact test

In the blind group, 6 patients could not be intubated on the first attempt. Among these 6 patients, 5 of them were successfully intubated on the second attempt, and the remaining 1 patient was successfully intubated on the third attempt.

In the blind group, two patients were assessed to have Modified Mallampati class III. Of these, one patient was successfully intubated on the first attempt after application of the second manoeuvre (up-down). The other patient was intubted successfully on the second attempt with the use of only the first manoeuvre (Extension), after one episode of oesophageal intubation. Both patients complained of mild sore throat postoperatively.

In the lightwand group, three patients had a Modified Mallampati Class III. Two patients were intubated on the first attempt without the need for any adjustment manoeuvres to the ILMA. The third patient was successfully intubated on the first attempt after application of the second manoeuvre (Up-down). There was no incidence of oesophageal intubation or postoperative adverse events in any of these three patients.

Table 3: Adjustment manoeuvres required to achieve successful intubation

\begin{tabular}{|c|c|c|c|}
\hline Parameters & Blind group n=30 & Lightwand group n=30 & p value \\
\hline None used & 16 & 21 & $0.288^{*} \mathrm{NS}$ \\
\hline $1^{\text {st }}$ manoeuvre Extension & 6 & 7 & $0.223^{*} \mathrm{NS}$ \\
\hline $2^{\text {nd }}$ manoeuvre Up-down & 6 & 2 & \\
\hline $3^{\text {rd }}$ manoeuvre Chandy & 2 & 0 & \\
\hline $\mathrm{X}^{2}$ test & \multicolumn{3}{|l}{} \\
\hline
\end{tabular}

In the blind group, 16 patients $(53 \%)$ could be intubated through the ILMA without utilising any manoeuvres. In the lightwand group, 21 patients $(70 \%)$ were intubated without any manoeuvres.
In the blind group, 14 patients required additional manoeuvres to achieve successful intubation. Among these, 6 patients could be successfully intubated using the first manoeuvre (Extension). Six patients required the use of the 
second manoeuvre (Up-down) to attain successful intubation. The third manoeuvre (Chandy) had to be used to achieve successful intubation in the remaining two patients. In the lightwand group, 9 patients required the use of adjustment manoeuvres. Seven of these 9 patients were successfully intubated using the first manoeuvre (Extension), and the remaining two patients with the second manoeuvre (Up-down). The need for the third manoeuvre (Chandy) did not arise in this group.

There was no statistical significance between the two groups with regards to number of manoeuvres applied to the ILMA. There was no incidence of desaturation to less than $95 \%$ in any of the 60 patients during the study. None of the intubation attempts proceeded beyond three minutes in any of the patients.

The presence of blood on the ILMA or on the TT was seen in a total of 7 patients in the blind group. Among these, in 4 patients blood was seen only on the ILMA, in 2 patients blood was on the ILMA and TT, and in 1 patient blood was present only on the TT. In the lightwand group, blood was seen on the ILMA in 6 patients. There was no evidence of blood on the TT in any of the patients in the lightwand group.

There was no statistically significant difference with respect to the presence of blood on the ILMA or TT between the two groups.

The incidence of sore throat and the nature of the sore throat were comparable in both the groups. Nine patients $(30 \%)$ in the blind group and 6 patients $(20 \%)$ in the lightwand group experienced a sore throat of a mild to moderate nature.

No patient sustained a severe sore thoat in either group. Five of the 15 patients who experienced a sore throat had evidence of blood on the ILMA and/ or TT. The overall incidence of sore throat due to intubation through the ILMA was $25 \%$.

Only 1 patient in the study, who belonged to the lightwand group, experienced a mild hoarseness of voice. This patient also had an associated mild sore throat. In this patient, intubation was successful on the first attempt without application of any manoeuvres, and there was no blood present on the ILMA or ETT.

No patient in the blind group complained of hoarseness of voice. There was no statistical difference between the two groups with regard to hoarseness of voice in the postoperative period.

\section{Discussion}

In our study, the ILMA was successfully inserted using a single handed rotational technique in all 60 patients. This compares favourably with previous studies ${ }^{[3]}$.

Tracheal intubation through the ILMA was successful in all the 60 patients in our study. The $100 \%$ success rate in intubation through the ILMA in our adult patients reinforces the importance of the ILMA as a conduit for tracheal intubation. Earlier studies show the success rate of intubation through ILMA to be about $90-100 \%{ }^{[2,4]}$.

We observed that with the use of the lightwand, tracheal intubation was successful on the first attempt in all 30 patients $(100 \%)$. In the blind group, intubation was successful on the first attempt in 24 patients $(80 \%)$. In the remaining 6 patients, the initial attempt resulted in oesophageal intubation. Among the 6 patients, 5 were successfully intubated on the second attempt and one patient required a third attempt, as the first 2 attempts were oesophageal intubations. This difference in first attempt success rate was statistically significant, indicating that the lightwand guided technique facilitates faster and more efficient intubation when compared to the blind technique.

In our study, the time taken for successful intubation in the lightwand group was marginally shorter compared to the time taken for intubation in the blind intubation group. It however, had a smaller standard deviation (5.68 seconds) with $60 \%$ of the intubations being achieved in 20 seconds or less. The intubation times in the blind group were more variable as indicated by a standard deviation of 21.09 seconds. In the blind group, $50 \%$ of the intubations were achieved in 20 seconds or less with the longest time for intubation being 125 seconds.

The results are comparable to a study done by Chan et al. who conducted a randomized comparison of intubation through the ILMA with and without the lightwand in 100 healthy adults. The success rate of intubation was $100 \%$ in both the groups. The blind intubation time was significantly longer than the lightwand guided intubation $(38.3 \pm 10.4 \mathrm{~s}$ versus $26.9 \pm 9.1 \mathrm{~s}, P<0.001)$.

Kihara et al. in their comparison between blind and lightwand guided tracheal intubation through the ILMA, in 120 anaesthetised and paralysed individuals, had a 93\% success rate of intubation in the blind group and $100 \%$ in the lightwand group ${ }^{[5]}$. They observed a significantly shorter intubation time in the lightwand group in comparison with the blind group (46 s versus $67 \mathrm{~s}$ ).

In another study done by Huerta MC et al. who conducted a randomized comparison of intubation through the ILMA with and without lightwand in 100 healthy adult patients, success rate of intubation was $100 \%$. The mean time taken for endotracheal intubation was higher in the group where patients were intubated blindly through the ILMA (38.3 \pm $10.4 \mathrm{~s}$ versus $26.4 \pm 9.1 \mathrm{~s}, \mathrm{P}<0.001)^{[6]}$.

Dimitriou et al. attempted flexible lightwand guided intubation via the $\mathrm{ILM}^{\mathrm{TM}}$ in 44 patients in whom direct laryngoscopy and intubation was unsuccessful ${ }^{[7]}$. They were able to intubate 38 patients $(86 \%)$ on the first attempt, 3 patients $(7 \%)$ on the second attempt, and 2 patients $(5 \%)$ on the third to fifth attempt. Intubation failed in one patient (2\%). This patient was subsequently woken up in view of persistent oesophageal intubation. During the course of this study, they conducted a parallel study on 48 patients with predicted difficult airways. They found that the lightwand guided intubation was successful in 48 of 48 (100\%) and 43 $(90 \%)$ of them were successful in the first attempt.

The adjustment manoeuvres chosen for our study were extension of the ILMA, the up-down movement and the Chandy manoeuvre. These were applied in a sequential manner. If the preceding manoeuvre was not successful, then the next manoeuvre was tried.

The first manoeuvre was the extension manoeuvre. Dimitriou et al. encouraged the use of this manoeuvre. It is considered to be helpful as, with its use, the cuff of the ILMA gets tilted anteriorly and slightly proximally. This may help in redirecting the TT away from the oesophagus to a more antero-superior target in the pharynx.

The second manoeuvre was the up-down technique. This manoeuvre is considered to be useful as it may randomly realign the position of the cuff in the pharynx to a more optimal location, it may change the position of the epiglottis or release a down folded epiglottis.

The third manoeuvre was the Chandy manoeuvre. This two 
step manoeuvre helps in first aligning the internal aperture of the device with the glottis opening. The second step, which involves a vertical lift, then prevents the tracheal tube from colliding with the arytenoids and facilitates the smooth passage of the TT into the trachea.

Sixteen patients in the blind group (53.3\%) were successfully intubated without any manoeuvres compared to 21 patients $(70 \%)$ in the lightwand group. In the blind group, of the 14 patients who required additional manoeuvres, 6 were successfully intubated using the $1^{\text {st }}$ manoeuvre (Extension), another 6 after applying the $2^{\text {nd }}$ manoeuvre (up-down) and the remaining 2 after the use of the third manoeuvre (Chandy). In the lightwand group, only 9 patients required additional manoeuvres, with 7 of them successfully intubated using the $1^{\text {st }}$ manoeuvre and 2 after use of the $2^{\text {nd }}$ manoeuvre. Although this difference between the number of manoeuvres used was not statistically significant, we observed that more number of patients required additional manoeuvres in the blind group. These findings were in agreement with earlier studies ${ }^{[2,6]}$.

By providing information regarding the location of the tip of the TT, lightwand guided intubation helps in avoiding oesophageal intubation. Absence of a glow near the cricothyroid membrane, or the initial glow disappearing during downward movement of the TT-lightwand assembly indicates oesophageal intubation. A bright glow in the middle at the level of the laryngeal prominence that continues with the downward movement of the TTlightwand assembly until the suprasternal notch indicates tracheal intubation.

Preceding studies also showed analogous results. Kihara et $a l$. showed no difference in postoperative pharyngolaryngeal morbidity between the two groups. Presence of blood on the ILMA or TT, which they took as a sign of mucosal injury, also occurred nearly equally in both groups. Chan et al. also concluded that there was no significant difference in the incidence of sore throat, hoarseness of voice and mucosal bleeding between the blind and lightwand guided intubation groups ${ }^{[8]}$.

Our study had few limitations. Firstly, it was not possible to achieve a double blinded study. Although the patients were randomly assigned to each group by a random number table, the intubator knew which group the patient belonged to. To minimize bias, an independent observer was employed to record the intubation time, however this observer too was not blinded. Only the third observer who evaluated postoperative sore throat and hoarseness of voice was blinded to the earlier events.

We had a sample size of 60 patients. This sample size may not be adequate to show a statistically significant difference in intubation times and the number of manoeuvres utilized. Further study with a bigger sample size may be able to demonstrate these differences. A crossover design where an unsuccessful attempt with one technique leads to the use of the alternative technique may help in providing the superiority of one technique over other.

As the ILMA had been intended for use in the difficult airway, it may have been advisable not to paralyse the patients during the study. Keeping the patients spontaneously breathing would have simulated a difficult airway scenario more aptly.

Our study did draw out some advantages of the lightwand guided technique over the blind technique of intubation through the ILMA. The lightwand guided technique proved to be easier than the blind technique for intubation. This is supported by a Kihara et al. who showed that there is no learning curve for the light guided tracheal intubation through the ILMA. Chan et al. also described the lightwand guided technique of intubation through the ILMA as a technique that is easy to learn ${ }^{[9]}$.

By providing information about the location of the ILMA, the lightwand guided technique of intubation through the ILMA allows for adjustment manoeuvres to be made prior to attempting intubation. This then decreases the time to successful intubation, decreases the number of adjustment manoeuvres required and prevents oesophageal intubation. ${ }^{10}$ Therefore, as per the evidence obtained from our study, which is corroborated by previous published studies, we conclude that the lightwand guided technique of intubation through the ILMA is efficient and safer than the blind technique of intubation through the ILMA. Postoperative pharyngolaryngeal morbidity is not increased with the use of the lightwand through the ILMA.

\section{Conclusion}

In anaesthetized, paralysed adult patients, the blind and lightwand guided techniques of intubation through the ILMA are comparable with respect to the rate of successful intubation, the time taken for successful intubation and postoperative pharyngolaryngeal morbidity, in the form of sore throat and hoarseness of voice. However, an advantage of the lightwand guided technique is that it effectively helps to avoid accidental oesophageal intubations.

\section{References}

1. A Choyce, MS Avidan, C Patel, A Harvey Comparison of laryngeal mask and intubating laryngeal mask insertion by the naïve intubator. $\mathrm{Br} \mathrm{J}$ Anaesth 2000;84:103-5.

2. Asai T, Wagle A U, Stacey M. Placement of the intubating laryngeal mask is easier than the laryngeal mask during manual in-line neck stabilization. $\mathrm{Br} \mathrm{J}$ Anaesth 1999;82,Issue5:712-4.

3. Ferson DZ, Rosenblatt WH, Johansen MJ, Osborn I, Ovassapian A. Use of the Intubating LMA-Fastrach ${ }^{\mathrm{TM}}$ in 254 Patients with Difficult-to-manage Airways. Anesthesiology. 2001; 95(5):1175-81. https:// doi.org/10.1097/00000542-200111000-00022 PMid:11684987.

4. Kapila A, Addy EV, Verghese C, Brain AIJ. The intubating laryngeal mask airway: an initial assessment of performance. Br J Anaesth 1997;79:710-3.

5. S Kihara, S Watanabe, $\mathrm{N}$ Taguchi. A comparison of blind and lightwand-guided tracheal intubation through the intubating laryngeal mask. Anaesthesia 2000;55:427-31

6. Huerta MC, Gil OFI, Aizpuru GAP, et al. A randomized comparison using intubation by means of Fastrach (ILMA) with and without lightwand (Trachlight). Trauma. 2006;9(1):6-11

7. Dimitriou V, Voyagis G, Brimacombe JR. Flexible lightwand-guided tracheal intubation with the intubating laryngeal mask Fastrach ${ }^{\mathrm{TM}}$ in adults after unpredicted failed laryngoscope guided intubation. Anaesthesiology 2002;96:296-9

8. Myatra SN, Shah A, Kundra P, Patwa A, Ramkumar V, Divatia JV, et al. All India Difficult Airway Association 2016 guidelines for the management of 
unanticipated difficult tracheal intubation in adults. Indian J Anaesth 2016;60:885-98.

9. Randa Badawi, Nashwa Nabil Mohamed, Mohamed Mohamed Abd Al Haq. Tips and tricks to increase the success rate of blind tracheal intubation through the Air- $Q^{\mathrm{TM}}$ versus the intubating laryngeal mask airway Fastrach $^{\mathrm{TM}}$. Egyptian Journal of Anaesthesia Volume 30, Issue 1, January 2014, Pages 59-65.

10. Navya Mishra, Avnish Bharadwaj. Comparison of Fiberoptic-Guided Tracheal Intubation Through Intubating Laryngeal Mask Airway (ILMA) FastrachTM and Ambu ${ }^{\circledR}$ Aura-i ${ }^{\text {TM: }}$ A Randomized Clinical Study. Cureus. 2020 Sep; 12(9): e10178. 